\title{
2013 ADSA Undergraduate Scholarship Recognition Awards
}

\author{
Sophomores \\ (Bronze certificate) \\ Amy McBirney \\ Suzanne Chickering \\ Heather Hunt \\ Quinn Kolar \\ Amanda Moretti \\ Jaime Mowry \\ Kirstin Torgerson \\ Meghan Vaill
}

Annette Llanes

George FitzPatrick

Isaac Haagen

Ashley Malawy

Alexander Tebbe

Alexis Thompson
Juniors

(Silver certificate)
Seniors

(Gold certificate)

\section{California Polytechnic State University; San Luis Obispo}

Katie Nutcher

Taylor Pires

Zachary Rios

\section{Cornell University}

Cassandra Chittenden

Rebecca Harrison

John Kelder

Emily Knight

Anna Laggis

Paul LaPierre

Elizabeth Martens

Zachary Smith

Matthew Sweeney

Katelyn Walley

Megan Wildman

Michigan State University

Courtney Doan

Madeleine Erba

Katelyn Horning

\section{North Carolina State University}

Ginger Hobgood

The Pennsylvania State University

Denise Beam

Dakota Grove

Caleb Grove

Brianne Rice

\section{University of Illinois}

Aaron Mitchell

Blaine Melody

\section{University of Kentucky}

Jillian Johnson

Ariel Waldeck 
AWARDS PROGRAM OF THE AMERICAN DAIRY SCIENCE ASSOCIATION

Virginia Polytechnic Institute and State University

Emma Reeves

Allison Sivak
Erin Klingensmith

Clayton Phelps

Roxanne Seltzer

Clayborne Zimmerman
Carissa Doody

Isaac Hammock

Sarah Williams 